\title{
HCC with portal vein tumor thrombosis: how to manage?
}

\author{
Naoki Kawagishi ${ }^{1,2}$ (1) \\ Received: 6 July 2020 / Accepted: 4 September 2020 / Published online: 19 September 2020 \\ (c) Asian Pacific Association for the Study of the Liver 2020
}

The existence of the portal vein tumor thrombus (PVTT) showed a terribly poor prognosis for the hepatocellular carcinoma (HCC) patients in the past decades. However, latest clinical studies elucidate that the hepatic resection introduces a good prognosis for some selected cases with less severe PVTT. Moreover, some adding treatment options, such as interventional therapies and systemic agents, could improve the overall survival of the patients with severe forms of PVTT.

Although the treatment of the HCC with PVTT is controversial due to the variety of the situations, the best survival is predicted with the hepatic resection [1-7]. So the hepatic resection should be considered at first for the patients with relatively preserved liver functions, and younger generations without extrahepatic metastasis (Fig. 1). If the patient is difficult with the surgical operation, such as too small remnant liver after resection, cardiac or respiratory distress for operation, poor performance status, and old generations, the other options should be selected. The cases with extensive tumor thrombus in the superior mesenteric vein or splenic vein accompanied with collaterals are also contraindications for the hepatic resection. The next step for the decision for the treatment is to clarify the classifications of the PVTT. The cases with up to second-order branch PVTT should be considered for transarterial chemoembolization (TACE) after the diagnosis of contraindications for hepatic resection. The patients with over first-order branch should be considered for hepatic arterial infusion chemotherapy (HAIC) or systemic therapy like sorafenib or newly arising agents. Without the consideration of the classification of the PVTT, stereotactic body radiotherapy (SBRT) or transarterial radioembolization (TARE) is the candidate for the treatment

Naoki Kawagishi

kawan@med.tohoku.ac.jp

1 Division of Transplant Surgery, JCHO Sendai Hospital, 3-16-1 Tsutsumi-machi, Aoba-ku, Sendai 981-8501, Japan

2 Division of General Medicine, Imakane Town National Health Insurance Hospital, 17-2 Azaimakane, Imakane 049-4318, Japan after the decision of withdrawing the hepatic resection. If the treatments, such as hepatic resection, TACE, SBRT, and TARE, could reduce the main tumor size and disappear of the PVTT, liver transplantation would be the candidate for the next step in some selected patients. And hepatic resection or radiofrequency ablation (RFA) after the downstaging by TACE, SBRT, TARE, and HAIC also would be possible for the selected cases. If the tumor has disappeared after the first treatment for the HCC with the PVTT, anti-HBV or anti-HCV therapy should be introduced aggressively. Even if it becomes Child-Pugh $\mathrm{C}$ after the first anti-cancer treatment, direct antiviral agent (DAA) therapy for $\mathrm{HCV}$ is possible [8]. Unfortunately, the patient whose liver reserve is Child-Pugh C does not have active treatment for the HCC with the PVTT so far.

In the following section, the promising clinical treatments for the patients of HCC with PVTT, mainly hepatic resection, are discussed by the countries or regions. In accordance with the randomized controlled SHARP trial, the European Association for the Study of the Liver due to the Barcelona Clinic Liver Cancer staging system recommends sorafenib as systemic chemotherapy for advanced HCC. And due to the American Association for the Study of the Liver Disease/ Barcelona Clinic for Liver Cancer (AASLD/BCLC) Staging System and the treatment guidelines, HCC with portal vein invasion, or PVTT, is considered as the terminal stage of the carcinoma in terms of the extremely short OS and disease free survival (DFS). The molecular targeting agents, sorafenib, lenvatinib, and regorafenib, are the only proposed treatment options for the HCC with portal invasion or PVTT patients. The MST of patients with advanced HCC treated with sorafenib is reported as short as 10.7 months. This means that only a modest survival benefit is received in these patients with the molecular targeting agents.

In spite of the European and USA proposal, Japanese nation-wide survey proposed the hepatic resection [3, 4]. Recent progress in the methodology of hepatic resection and pre- and post-operative management, the surgical approach for the patients of HCC with PVTT has changed to the reasonable trusted treatment choice with an acceptable 
Fig. 1 Simplified algorithm of the proposed management of the HCC with PVTT except extrahepatic metastasis. PVTT portal vein tumor thrombosis, TACE transarterial chemoembolization, SBRT stereotactic body radiotherapy, TARE transarterial radioembolization, HAIC hepatic arterial infusion chemotherapy, $R F A$ radiofrequency ablation

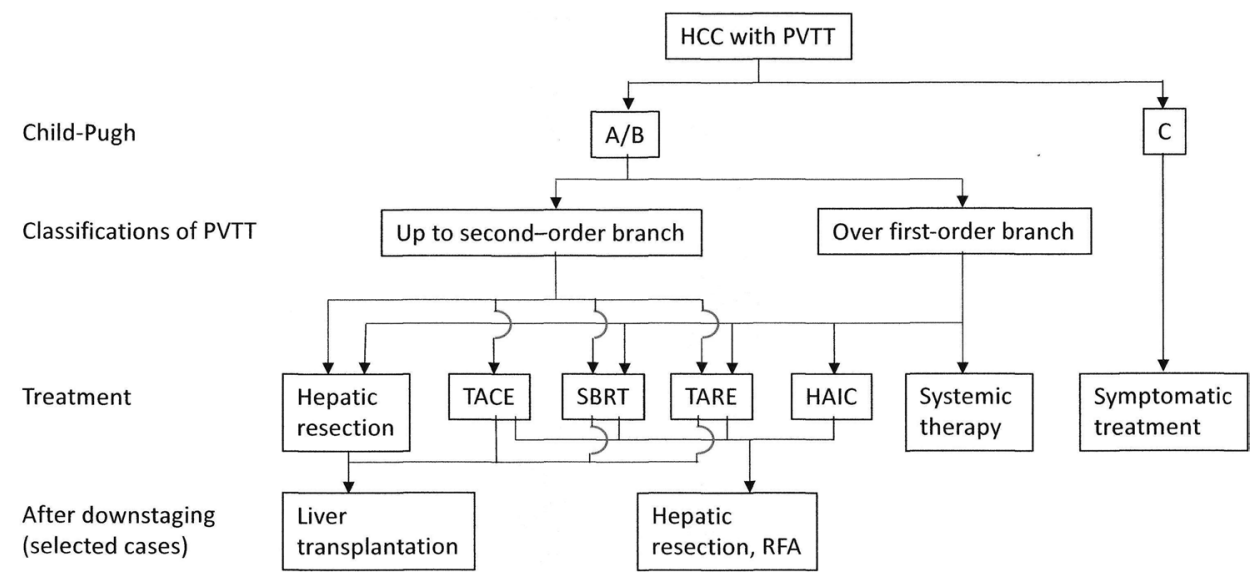

mortality and morbidity rate. Several aggressive surgeons reported that sophisticated surgical techniques were applied for the patients of HCC with PVTT, and they acquired good results [4]. Nevertheless the better results, the number of patients registered in these studies was relatively small, and the studies had significant selection bias [4]. Although the overall survival was not completely satisfied, another Japanese institute reported that the hepatic resection for the patients of HCC with PVTT offered the long-term prognosis in some selected patients [3]. They also suggested a prognostic index composed of the existence of ascites, prothrombin time, and maximal diameter of the tumor, based on analyzing the prognostic factors for patients with PVTT in the major portal vein [3]. In case of the treatment for the patients of the HCC associated with a major portal vein invasion, the prognostic index might be useful to choose the adequate treatment modalities. However, it might have happened that the immediate multiple intrahepatic recurrences after the hepatic resection worsened the OS in the patients of HCC with PVTT. Therefore, to avoid the early multiple intrahepatic recurrences, the adjuvant HAIC has been introduced after the resection of HCC with PVTT in some institutions [3].

The other study in Japan was to clarify the impact of hepatic resection for HCC with PVTT in the major portal vein in a larger cohort [4]. They conducted the retrospective multi-institutional study of the patients who underwent hepatic resection for HCC with major PVTT after applying the propensity score-based matching. And they concluded that hepatic resection is expected to have a longer OS than non-surgical treatment in HCC patients with PVTT. They also concluded that if the PVTT is expanded within the firstorder branch, hepatic resection should be the first treatment option, particularly for the patients with good liver functions. The other authors also described that the macroscopic curative hepatic resection was beneficial for the patients of HCC with PVTT, even with tumor thrombi in the first branch or trunk of the portal vein [3]. Moreover, they recommended the adjuvant postoperative HAIC to improve the disease-free survival (DFS) and OS in such patients.

In Korea, resulting from the multicenter studies, hepatic resection, as a first treatment option, indicated the better long-term OS than TACE or sorafenib for resectable HCC patients with type I PVTT (tumor thrombi involving the segmental branches of the portal vein or above) and good hepatic functions [5]. As a result, they suggested that treatment strategies for HCC with PVTT should not be limited to the molecular targeting drugs such as sorafenib, lenvatinib, and regorafenib. To prolong the OS of the patients with advanced BCLC stage HCC, the choice of the treatment method should be selected in accordance with the Child-Pugh class, the extent of PVTT, and the location of HCC.

In Taiwan, they concluded that hepatic resection for the patients of HCC with PVTT provides significantly better long-term OS than TACE under the population of the unselected patients or the patients adjusted using the propensitybased model [6]. Therefore, hepatic resection is the first of choice for the treatment of the patients of HCC with PVTT. In mainland China, they also concluded the superiority of the hepatic resection for the patients of HCC with PVTT in an article in this issue [7] on which this Editorial has been written.

In terms of the OS, the results of the western countries were poorer than that of east Asian countries [9, 10]. In Japan, two multicenter studies [3, 4] reported that the MST of the non-hepatic resection patients was 1.77 years shorter than that of the hepatic resection patients (1.10 years vs. 2.87 years; $p<0.001)$ and 0.88 years shorter than that of the hepatic resection patients (1.57 years vs. 2.45 years; $p<0.001)$ in a propensity score-matched cohort. And the subgroup analysis elucidated that the hepatic resection presented a survival benefit for the patients in spite of the patient's age, etiology of HCC, elevation of the tumor marker, and the number of tumors. And the other study reported that MST was 21.5 months and the 5-year OS 
was $25.7 \%$. The MST of the non-HAIC group was shorter than that of HAIC group (18.7 months vs. 28.1 months, $p=0.0024)$. The level of the protein induced by vitamin $\mathrm{K}$ antagonist-II (PIVKA-II) (DCP: des-gamma carboxyprothrombin), the diameter of the tumor, and the treatment of adjuvant HAIC were the significant prognostic factors for OS.

In terms of the single center report, a report from the Japanese center [11] concluded the excellent results. Their cumulative 5-year OS (5yrOS) and 5-year DFS (5yrDFS) rates of the 372 patients were $58.3 \%$ and $31.3 \%$, respectively. And the subgroup analysis elucidated that the OS of the patients with macroscopic PVTT (vp2-4) who had preoperative TACE was comparable to that of patients who did not have TACE $(p=0.747)$. They also reported that only alpha-fetoprotein (AFP) $>100 \mathrm{mg} / \mathrm{dL}$ was independently associated with DFS in the subgroup analysis of stage II-III HCC $(n=148)$.

The other treatment modalities for the HCC with PVTT include TARE, liver transplantation after prior downstaging with TACE, and SBRT $[12,13]$. These multimodality treatments improved the survival and the quality of life for some patients. And clinical trials are going towards molecular therapies that target the points of the HCC carcinogenesis [13].

The difference of the treatment results for the patients of HCC with PVTT is dependent on multiple factors, so it is difficult to elucidate the reason why the difference exists among each country. Chen $\mathrm{ZU}$ et al. [7] demonstrated that in mainland China "3-year survival" was defined as "long-term survival"; however, "3-year survival for HCC with PVTT patients" was not long for the Japanese, Korean, and Taiwanese experiences. The authors described the several limitations of this article in terms of the selection biases, lack of standardization in the operation, and etiology of HCC. These factors should be elucidated in future in mainland China.

The treatment modalities for HCC with PVTT represent surgical resection, HAIC, TACE, TARE, SBRT, molecular targeting drugs, RFA and liver transplantation. The indication of the ideal therapy for each patient needs multi-disciplinary consideration. Future study should be established for improving the overall survival of the HCC patients with PVTT.

\section{Compliance with ethical standards}

Conflict of interest Naoki Kawagishi declares that he has no conflict of interest.

Informed consent All procedures followed were in accordance with the ethical standards of the responsible committee on human experimentation (institutional and national) and with the Helsinki Declaration of 1975, as revised in 2008 (5). Informed consent was obtained from all patients for being included in the study.

Animal studies This article does not contain any studies with animal subjects.

\section{References}

1. Minagawa M, Makuuchi M. treatment of hepatocellular carcinoma accompanied by portal vein tumor thrombosis. World J Gastroenterol. 2006;12(47):7561-7.

2. Thomas MB, Jaffe D, Choti MM, Belghiti J, Curley S, Fong Y, et al. Hepatocellular carcinoma: consensus recommendations of the national cancer institute clinical trials planning meeting. J ClinOncol. 2010;28:3994-4005.

3. Hatano E, Uemoto S, Yamane H, Yamamoto M, Japanese society of hepato-biliary-pancreatic surgery. Significance of hepatic resection and adjuvant hepatic arterial infusion chemotherapy for hepatocellular carcinoma with portal vein tumor thrombus in the first branch of portal vein and the main portal trunk: a project study for hepatic surgery of the Japanese society of hepato-biliary-pancreatic surgery. J HepatobiliaryPancreatSci. 2018;25:395-402.

4. Kokudo T, Hasegawa K, Matsuyama Y, Takayama T, Izumi N, Kadoya M, et al. Survival benefit of liver resection for hepatocellular carcinoma associated with portal vein invasion. J Hepatology. 2016;65:938-43.

5. Lee JM, Jang BK, Lee YJ, Choi WY, Choi SM, Chung WJ, et al. Survival outcomes of hepatic resection compared with transarterial chemoembolization or sorafenib for hepatocellular carcinoma with portal vein tumor thrombosis. ClinMolHepatol. 2016;22:160-7.

6. Liu PH, Lee YH, Hsia CY, Hsu CY, Huang YH, Chiou YY, et al. Surgical resection versus transarterial chemoembolization for hepatocellular carcinoma with portal vein tumor thrombosis: a propensity score analysis. Ann SurgOncol. 2014;21:1825-33.

7. Chen ZH, Zhang XP, Lu YG, Li LQ, Chen MS, Wen TF, et al. Actual long-term survival in $\mathrm{HCC}$ patients with portal vein tumor thrombus after liver resection: a nationwide study. HepatolInt. 2020. https:// doi.org/10.1007/s12072-020-10032-2(Online ahead of print).

8. El-Sherif O, Jiang ZG, Tapper EB, Huang KC, Zhong A, Osinusi A, et al. Baseline factors associated with improvements in decompensated cirrhosis after direct-acting antiviral therapy for hepatitis C virus infection. Gastroenterology. 2018;154:2111-21.

9. Llovet JM, Ricci S, Mazzaferro V, Hilgard P, Gane E, Blanc JF, et al. Sorafenib in advanced hepatocellular carcinoma. N Engl J Med. 2008;359:378-90.

10. Bruix J, Raoul JL, Sherman M, Mazzaferro V, Bolondi L, Craxi A, et al. Efficacy and safety of sorafenib in patients with advanced hepatocellular carcinoma: subanalyses of a phase III trial. J Hepatol. 2012;57:821-9.

11. Yamamoto Y, Ikoma H, Morimura R, Shoda K, Konishi H, Murayama Y, et al. Post-hepatectomy survival in advanced hepatocellular carcinoma with portal vein tumor thrombosis. World J Gastroenterol. 2015;21:246-53.

12. Lau WY, Sangro B, Chen PJ, Cheng SQ, Chow P, Lee RC, et al. Treatment for hepatocellular carcinoma with portal vein tumor thrombosis: the emerging role for radioembolization using yttrium-90. Oncology. 2013;84:311-8.

13. Couri T, Pillai A. Goals and targets for personalized therapy for HCC. HepatolInt. 2019;13:125-37.

Publisher's Note Springer Nature remains neutral with regard to jurisdictional claims in published maps and institutional affiliations. 